\title{
THE ALGEBRAIC CHARACTER OF A CLASS OF HAR- MONIC FUNCTIONS IN THREE VARIABLES ${ }^{1}$
}

\section{P. M. PEPPER}

1. Introduction. Let the harmonic function in three variables $x, y, z$ be given by its series development into spherical harmonics, that is, let the harmonic function be given by

$$
F(r, \cos \theta, \phi)=\sum_{n=0}^{\infty} \sum_{n=-n}^{n} A_{n \nu} r^{n} P_{n}^{(v)}(\cos \theta) e^{i \nu \phi},
$$

where spherical coordinates have been introduced by the transformation

$$
x=r \cos \theta, \quad y=r \sin \theta \cos \phi, \quad z=r \sin \theta \sin \phi .
$$

It is obvious that the coefficients $A_{n}$ of the series development (1) determine completely the function $F(r, \cos \theta, \phi)$. Thus the sequence of coefficients determines the analytic character of $F$, the locations and nature of singular points, and so on. It seems therefore natural to look for and extract those properties of a sequence of coefficients $A_{n v}$ which most readily yield relevant information. In the case of harmonic functions of two variables, the problem reduces immediately to that of detecting the singularities of an analytic function given by its Taylor development. This last mentioned problem, also known as the Hadamard problem, has occupied the attention of mathematicians for a considerable period and a great many results have been obtained. When dealing, however, with harmonic functions of three variables, the problem becomes much more difficult and it seems impossible to apply directly the methods developed in the theory of functions of one complex variable. We have, however, at our disposal the method of integral operators introduced by S. Bergman, which enables us to represent harmonic functions in three variables by means of an integral operator on a function of a complex variable. This representation makes it possible to carry over certain results of the well known theory of functions of one complex variable into the relatively little developed theory of harmonic functions of three variables. The present paper is to be considered as a part of an extended program of study of harmonic functions given by the de-

Presented to the Society, November 29, 1947, under the title Algebraic harmonic functions; received by the editors July 23, 1948 and, in revised form, October 4, 1948.

${ }^{1}$ Paper done under contract with the Office of Naval Research N5ori 76-16NRo43-046 at Harvard University. 
velopment (1) through the above indicated means.

In the present paper we obtain sufficient conditions for (1) to represent an algebraic function, taking as a starting point the following result of $S$. Bergman [1].2

Let $g(u, \zeta)$ be a rational function in two variables $u$ and $\zeta$, and, putting

$$
u=x+i y\left(\frac{\zeta+\zeta^{-1}}{2}\right)+z\left(\frac{\zeta-\zeta^{-1}}{2}\right)
$$

define $H(x, y, z)$ by the integral

$$
H(x, y, z)=\frac{1}{2 \pi i} \int_{c} g(u, \zeta) d \zeta
$$

where $C$ is a suitable curve in the complex $\zeta$-plane. Then $H(x, y, z)$ expressed as a function of $r, \cos \theta, \phi$ through (2) represents an algebraic harmonic function $F(r, \cos \theta, \phi)$, and, conversely, every algebraic harmonic function can be obtained from (4) with $g(u, \zeta)$ algebraic or an integral of a specified kind of an algebraic function.

Moreover, if by means of $(3), g(u, \zeta)$ is written as the quotient of two polynomials in $x, y, z$, and $\zeta$ without common factors

$$
g(u, \zeta)=\frac{p(u, \zeta)}{q(u, \zeta)}=\frac{P(\zeta ; x, y, z)}{Q(\zeta ; x, y, z)},
$$

then the degree of $Q(\zeta ; x, y, z)$ in $\zeta$ gives, in general, the multivaluedness of the algebraic function $H(x, y, z)=F(r, \cos \theta, \phi)$, that is, the number of space sheets which are necessary in order to make it uniform.

The author wishes to thank Dr. A. Dvoretzky and Dr. M. Schiffer for their helpful suggestions.

2. Criteria for rationality of functions of two variables. In view of the foregoing it is important for us to find conditions on the coefficients $a(n, \nu)$ in the double series development of $g(u, \zeta)$,

$$
g(u, \zeta)=\sum_{n=0}^{\infty} \sum_{\nu=-\infty}^{\infty} a(n, v) u^{n} \zeta^{\nu}
$$

which entail the rationality of $g(u, \zeta)$ in both its arguments.

Toward this end it will be found convenient to introduce the following notations. For any sequence $\left\{a_{n}\right\}(n=0,1,2, \cdots)$ we shall, using the corresponding capital letter, employ the customary nota-

2 Numbers in brackets refer to the references cited at the end of the paper. 
tion

$$
A_{\lambda}^{(\mu)}=\left|\begin{array}{llll}
a_{\lambda} & a_{\lambda+1} & \cdots & a_{\lambda+\mu} \\
\vdots & & & \\
a_{\lambda+\mu} & a_{\lambda+\mu+1} & \cdots & a_{\lambda+2 \mu}
\end{array}\right| ; \lambda, \mu=0,1,2, \cdots
$$

for the Kronecker determinants.

Furthermore, if $a(n, \nu)(n, \nu=\cdots,-1,0,1,2, \cdots)$ is a double sequence, we again use the corresponding capital letter in denoting the following determinants:

$$
\begin{aligned}
A_{\lambda}^{(\mu)}\left(k_{0}, k_{1}, \cdots, k_{\mu}\right) & \\
= & \left|\begin{array}{lll}
a\left(\lambda, k_{0}\right) & a\left(\lambda+1, k_{1}\right) & \cdots a\left(\lambda+\mu, k_{\mu}\right) \\
\vdots & & \\
a\left(\lambda+\mu, k_{0}\right) & a\left(\lambda+\mu+1, k_{1}\right) & \cdots a\left(\lambda+2 \mu, k_{\mu}\right)
\end{array}\right|,
\end{aligned}
$$

$\lambda=\cdots,-1,0,1, \cdots ; \mu=0,1,2, \cdots ;$ and $k_{0}, k_{1}, \cdots, k_{\mu}$ being any $\mu+1$ integers.

Since our aim in this paper is merely to derive certain sufficient conditions for the coefficients of (1) in order that it represent an algebraic harmonic function, it will be enough for us to confine ourselves to functions $g(u, \zeta)$ which have the series development

$$
g(u, \zeta)=\sum_{n=0}^{\infty} \sum_{\nu=n}^{n} a(n, v) u^{n} \zeta^{\nu} .
$$

Thus, whenever employing some notation such as (6) where coeffcients $a(n, \nu)$ with $|\nu|>n$ appear, we invariably use the convention

$$
a(n, \nu)=0 \quad \text { for }|\nu|>n .
$$

The curve of integration in (4) will surround the origin and hence the series (7) has to be convergent for a sufficiently small $|u|$ and $\zeta$ on the curve. Indeed, in order to manipulate freely with the series, it is sufficient that it converge uniformly and absolutely. This curve may be taken as a circle about the origin in the $\zeta$-plane so that the Cauchy-Hadamard criterion for uniform and absolute convergence applies. If there exists a positive and finite $r$ such that

$$
\limsup _{n \rightarrow \infty}\left(\sum_{r=-n}^{n}|a(n, v) r|\right)^{1 / n}<\infty,
$$

then the series (7) is absolutely and uniformly convergent for $|\zeta|=r$ and $|u|$ sufficiently small. There is, of course, no loss of generality 
in assuming $r=1$, and in this case inequality (9) becomes lim sup $\left(\sum_{\nu--n}^{n}|a(n, \nu)|\right)^{1 / n}<\infty$. This condition is equivalent to: there exists a finite $R$ such that

(10) $|a(n, \nu)|<R^{n}$ for $\nu=-n,-n+1, \cdots, n ; n=0,1,2, \cdots$.

To establish rationality of $g(u, \zeta)$ in both its arguments we establish it for $u$ and $\zeta$ separately by means of the well known criteria first given by Kronecker [3].

If we put

$$
c_{n}=c_{n}(\zeta)=\sum_{n=-n}^{n} a(n, \nu) \zeta^{\nu}
$$

in (7), the condition for the rationality of $g(u, \zeta)$ in $u$ becomes the Kronecker condition for the series $\sum_{n=0}^{\infty} c_{n} u^{n}$ to represent a rational function. One of the conditions given by Kronecker is that the determinants $C_{0}^{(\mu)}$ vanish for all sufficiently large $\mu$.

Now, on substituting the values of $c_{n}$ as given by (11) in $C_{\lambda}^{(\mu)}$ we obtain

$$
C_{\lambda}^{(\mu)}=\left|\begin{array}{llll}
\sum_{\nu=-\lambda}^{\lambda} a(\lambda, \nu) \zeta^{\nu} & \sum_{\nu=\lambda-1}^{\lambda+1} a(\lambda+1, \nu) \zeta^{\nu} & \ldots & \sum_{\nu=-\lambda-\mu}^{\lambda+\mu} a(\lambda+\mu, \nu) \zeta^{\nu} \\
\vdots & & & \\
\sum_{\nu=-\lambda-\mu}^{\lambda+\mu} a(\lambda+\mu, \nu) \zeta^{\nu} & \sum_{\nu=-\lambda-\mu-1}^{\lambda+\mu+1} a(\lambda+\mu+1, \nu) \zeta^{\nu} & \ldots & \sum_{\nu=-\lambda-2 \mu}^{\lambda+2 \mu} a(\lambda+2 \mu, \nu) \zeta^{\nu}
\end{array}\right| .
$$

Using the convention (8) and the notations (6) we easily see that

$$
C_{\lambda}^{(\mu)}=\sum_{m=-(1+\mu)(\lambda+\mu)}^{(1+\mu)(\lambda+\mu)} \sum_{k_{0}+k_{1}+\cdots+k_{\mu}=m} A_{\lambda}^{(\mu)}\left(k_{0}, k_{1}, \cdots, k_{\mu}\right) \zeta^{m}
$$

where the inner summation is taken over all integral values which add up to $\mathrm{m}^{3}$ Because of the linear independence of the powers of $\zeta$, the requirement that $C_{\lambda}^{(\mu)}$ vanish identically in $\zeta$ is evidently equivalent to the vanishing of the coefficients of the different powers of $\zeta$ in (12).

If we introduce the abbreviation

$$
A_{\lambda}^{(\mu)}\{m\}=\sum_{k_{0}+k_{1}+\cdots+k_{\mu}(\mu)=m} A_{\lambda}^{(\mu)}\left(k_{0}, k_{1}, \cdots, k_{\mu}\right),
$$

${ }^{3}$ From relation (8) it is seen that the $k_{\nu}$ may be restrained to satisfy $\left|k_{\nu}\right| \leqq \lambda+\mu+\nu$ without loss of generality, for, any $A_{\lambda}^{(\mu)}\left(k_{0}, k_{1}, \cdots, k_{\mu}\right)$ with $\left|k_{\nu_{0}}\right|>\lambda+\mu+\nu_{0}$ has a column of zeros. 
this condition can be written

$$
\begin{gathered}
A_{\lambda}^{(\mu)}\{m\}=0 \\
\text { for } m=-(\mu+1)(\lambda+\mu),-(\mu+1)(\lambda+\mu)+1, \cdots,(\mu+1)(\lambda+\mu) .
\end{gathered}
$$

Because of (8), all the sums appearing in (13) are finite.

We have thus established the following lemma.

LEMMA I. A necessary and sufficient condition for $g(u, \zeta)$ to be $a$ rational function of $u$ is that the series development satisfies the conditions $A_{0}^{(\mu)}\{m\}=0$ for $m=-\mu^{2}-\mu,-\mu^{2}-\mu+1, \cdots, \mu^{2}+\mu$ and all sufficiently large $\mu$.

We are, however, also interested in the degrees of the numerator and denominator of $g(u, \zeta)$ and for this purpose it is better to apply another form of the Kronecker criterion. We refer to the one stating that for $\sum c_{n} u^{n}$ to represent a rational function with the denominator of degree not exceeding $\beta$ and the numerator of degree not exceeding $\alpha(\alpha \geqq \beta-1),{ }^{4}$ it is necessary and sufficient that

$$
C_{\lambda}^{(\beta)}=0 \quad \text { for } \lambda=\alpha-\beta+1, \alpha-\beta+2, \cdots .
$$

Using in this criterion (12) and (13) we obtain the following lemma.

Lemma II. $A$ necessary and sufficient condition for $g(u, \zeta)$ to be a rational function of $u$ with the denominator of degree not exceeding $\beta$ and the numerator of degree not exceeding $\alpha$ is that

$$
A_{\lambda}^{(\beta)}\{m\}=0
$$

for $m=-(\beta+1)(\lambda+\beta),-(\beta+1)(\lambda+\beta)+1, \cdots,(\beta+1)(\lambda+\beta)$ and $\lambda=\alpha-\beta+1, \alpha-\beta+2, \cdots$.

In order to apply directly the Kronecker criteria in studying the rationality of $g(u, \zeta)$ in $\zeta$, it is convenient to introduce a n'ew variable $U$ through the substitution $u=U \zeta$. Simultaneous rationality in $u$ and $\zeta$ is, of course, equivalent to simultaneous rationality in $U$ and $\zeta$. We then have

$$
g(u, \zeta)=G(U, \zeta)=\sum_{n=0}^{\infty} \sum_{\nu=-n}^{n} a(n, \nu) U^{n} \zeta^{\nu+n}
$$

4 The restriction $\alpha \geqq \beta-1$ in the Kronecker criterion is not essential. This may readily be shown by his methods if terms $c_{n}=0$ are defined for all $n<0$. Henceforth, this restriction is omitted, and $C_{\lambda}^{(\mu)}$ with $\lambda<0$ are employed. 
Under the convergence conditions (9) and (10) imposed on the series $g(u, \zeta)$ it is possible to rearrange $G(U, \zeta)$ into

$$
G(U, \zeta)=\sum_{n=0}^{\infty} \sum_{\nu=n^{\prime}}^{\infty} a(\nu, n-\nu) U^{\nu} \zeta^{n}
$$

where $n^{\prime}=[(n+1) / 2]$, the brackets denoting, as customary, the integral part.

Putting

$$
b_{n}=b_{n}(U)=\sum_{\nu=n^{\prime}}^{\infty} a(\nu, n-\nu) U^{v} ; \quad n=0,1,2, \cdots,
$$

we have

$$
G(U, \zeta)=\sum_{n=0}^{\infty} b_{n} \zeta^{n}
$$

and the Kronecker condition for rationality becomes $B_{0}^{(\mu)}=0$ for all sufficiently large $\mu$.

We now put $a^{*}(n, \nu)=a(\nu, n-\nu)$ and denote by $A_{\lambda}^{*(\mu)}\left(k_{0}, k_{1}, \cdots, k_{\mu}\right)$ and $A_{\lambda}^{*(\mu)}\{m\}$ the quantities obtained from $A_{\lambda}^{(\mu)}\left(k_{0}, k_{1}, \cdots, k_{\mu}\right)$ and $A_{\lambda}^{(\mu)}\{m\}$ on replacing $a(n, \nu)$ by $a^{*}(n, \nu)$ in (6) and (13).

A calculation similar to the one that led to (12) gives

$$
B_{\lambda}^{(\mu)}=\sum_{m=\theta^{\prime}}^{\infty} \cdot A_{\lambda}^{*(\mu)}\{m\} U^{m}
$$

where

$$
s=s(\lambda, \mu)=(\lambda+\mu)(\mu+1) \text { and } s^{\prime}=s^{\prime}(\lambda, \mu)=[(s+1) / 2] .
$$

It should be remarked that if $k_{0}+k_{1}+\cdots+k_{\mu}<s^{\prime}$, then $A_{\lambda}^{*}(\mu)\left(k_{0}, k_{1}, \cdots, k_{\mu}\right)=0$ as a consequence of (8), for in that case each of the products in the complete expansion of the determinant has at least one zero factor. ${ }^{5}$

${ }^{5}$ Suppose that $a^{*}\left(\lambda+\nu_{0}, k_{0}\right) a^{*}\left(\lambda+1+\nu_{1}, k_{1}\right) \cdots a^{*}\left(\lambda+\mu+\nu_{\mu}, k_{\mu}\right)$ is a nonvanishing term in the complete expansion, then $\nu_{0}, \nu_{1}, \cdots, \nu_{\mu}$ is a permutation of $0,1, \cdots$, $\mu$. From (8) and $a^{*}(n, \nu)=a(\nu, n-\nu)$ it follows that $a^{*}(n, \nu) \neq 0$ only if $\nu \geqq n / 2$. When this is applied to the determinant term, we see that $k_{0} \geqq\left(\lambda+\nu_{0}\right) / 2, k_{1} \geqq\left(\lambda+1+\nu_{1}\right) / 2$, $\cdots, k_{\mu} \geqq\left(\lambda+\mu+\nu_{\mu}\right) / 2$. Addition of these inequalities yields $k_{0}+k_{1}+\cdots+k_{\mu}$ $\geqq(\lambda+\mu)(\mu+1) / 2$. The left member of this inequality is an integer and the right member is $s^{\prime}$ except when $\lambda$ is odd and $\mu$ is even, in which case it is $s^{\prime}-1 / 2$. Since $s^{\prime}$ is an integer, also, this implies that $k_{0}+k_{1}+\cdots+k_{\mu}$ is at least as great as $s^{\prime}$ even in this case. This permits $s^{\prime}$ to be introduced as a lower bound of the integers $m$ for which the test of Lemma III (below) must be applied, all smaller values being irrelevant. 
Thus, taking into account the convergence conditions (9) and (10) we may formulate the following lemma.

LEMma III. A necessary and sufficient condition for $G(U, \zeta)$ to be a rational function in $\zeta$ is that the coefficients $a(n, \nu)$ satisfy

$$
A_{0}^{*(i)}\{m\}=0 \quad \text { for integral } m \geqq\left(\mu^{2}+\mu\right)^{\prime}=\left[\left(\mu^{2}+\mu+1\right) / 2\right]
$$

and all sufficiently large $\mu$.

Again, when trying to obtain the degrees of the numerator and denominator it is useful to employ the other Kronecker criterion and thus obtain

LEMMA IV. A necessary and sufficient condition for $G(U, \zeta)$ to be rational in $\zeta$ with degree of denominator not exceeding $\delta$ and degree of numerator not exceeding $\gamma$ (cf. footnote 2 ) is that

$$
A_{\lambda}^{*(\delta)}\{m\}=0
$$

for integral $m \geqq s^{\prime}(\lambda, \delta) ; \lambda=\gamma-\delta+1, \gamma-\delta+2, \cdots$, where the function $s^{\prime}$ is given by (16). In particular, the truth of (18) for sufficiently large $\lambda$ is a necessary and sufficient condition on $G(U, \zeta)$ that its denominator be of degree not greater than $\delta$ in $\zeta$.

3. Application to harmonic functions. On taking the path of integration in (4) as, say, the circle $|\zeta|=1$, the term $a(n, \nu) u^{n} \zeta^{\nu}$ will for $|\nu| \leqq n$ yield $A_{n,} r^{n} P_{n}^{(\nu)}(\cos \theta) e^{i v \phi}$ with $A_{n \nu}=a(n, \nu) C_{2 n, n+\nu} i^{\nu} / 2^{n}$, where $C_{2 n, n+}$ denotes the binomial coefficient. From the fact that the integral operators operating on a rational function yield algebraic harmonic functions, we obtain the following theorem.

THEOREM I. Let the series (1) of spherical harmonics satisfy for some finite $R$

$$
\left|A_{n \nu}\right|<R^{n} \quad \text { for } \nu=-n,-n+1, \cdots, n ; n=1,2,3, \cdots,
$$

and, on putting

$$
\begin{array}{lrl}
a(n, \nu)=\frac{2^{n}(n+\nu) !(n-\nu) !}{(2 n) ! i^{\nu}} A_{n \nu} & \text { for }|\nu| \leqq n, \\
a(n, \nu)=0 & \text { otherwise, }
\end{array}
$$

let the following conditions be satisfied:

$$
A_{0}^{(\mu)}\{m\}=0 \quad \text { for } m=-\mu^{2}-\mu,-\mu^{2}-\mu+1, \cdots, \mu^{2}+\mu
$$


and

$$
A_{0}^{*(\mu)}\{m\}=0 \quad \text { for integral } m \geqq\left[\left(\mu^{2}+\mu+1\right) / 2\right]
$$

for all $\mu$ sufficiently large. Then $F(r, \cos \theta, \phi)$ is an algebraic function.

The condition (19) is easily seen by (20) to be the counterpart of assumption (10) which allowed us to rearrange the series, integrate term by term, and so on.

Using Lemmas II and IV, and the last results of Bergman mentioned in the introduction we can obtain some more detailed information. If $\alpha$ and $\beta$ are the degrees of numerator and denominator of $G(U, \zeta)$ in $U$ and $\gamma$ and $\delta$ the respective degrees in $\zeta$, then, since $U \zeta^{2}$ is a polynomial in $x, y, z$, and $\zeta$ of degree 2 in $\zeta$, it follows that the degree in $\zeta$ of $Q(\zeta ; x, y, z)$ of $(5)$ is at most equal to $2 \max (\alpha, \beta)+\delta$. (Cf. (15).) Thus we obtain the following theorem.

THEOREM II. Let the series of spherical harmonics (1) satisfy the condition (19) and furthermore, let there exist non-negative integers $\alpha, \beta, \delta$ such that the $a(n, \nu)$ defined by (20) satisfy

$$
\begin{gathered}
A_{\lambda}^{(\beta)}\{m\}=0 \\
\text { for } m=-(\beta+1)(\lambda+\beta),-(\beta+1)(\lambda+\beta)+1, \cdots,(\beta+1)(\lambda+\beta) ; \\
\lambda=\alpha-\beta+1, \alpha-\beta+2, \cdots, \text { and } \\
A_{\lambda}^{*(\delta)}\{m\}=0
\end{gathered}
$$

for integral $m \geqq s^{\prime}(\lambda, \delta)$ (cf. (16)) for all sufficiently large $\lambda$, then $F(r, \cos \theta, \phi)$ is an algebraic function which is at most $(2 \max (\alpha, \beta)+\delta)$ valued.

4. Remarks. The results of this paper may be extended and developed in several directions.

The coefficients $A_{n}$ of (1) are obtained directly from those coefficients of $\sum a(n, \nu) u^{n} \xi^{\nu}$ for which $|\nu| \leqq n$, and that is why we limited ourselves to the consideration of series $g(u, \zeta)$ which satisfy $a(n, \nu)$ $=0$ for $|\nu|>|n|$. However, the conditions of rationality of power series depend on all their coefficients and thus it would be interesting to find out not only when (7) represents a rational function but also when it can be extended, by introducing terms with $|\nu|>n$ into doubly infinite series representing rational functions. This would give less strict sufficient conditions for the algebraic character of (1).

These conditions would, however, be still very far from necessary and it would be interesting to derive from Bergman's result men- 
tioned in the introduction some necessary conditions for the algebraic character of (1).

Another direction in which it is possible to extend our results is to try to carry over through the integral operators into the theory of harmonic functions more detailed results of the theory of power series, that is, those of Hadamard, Mandelbrojt, Dvoretzky, and others relating to the location and nature of the singularities of analytic functions of one complex variable, into the theory of harmonic functions of three variables.

Finally, the method of integral operators has been extended by Bergman [3] to much more general partial differential equations than the Laplacian and thus it is possible to extend our results on harmonic functions to the solutions of these general partial differential equations.

\section{BIBLIOGRAPHY}

1. S. Bergman, Zur Theorie der algebraischen Potentialfunktionen der dreidimensionalen Raumes I and II, Math. Ann. vol. 99 (1928) pp. 625-659 and vol. 101 (1929) pp. 534-558.

2. - Classes of solutions of linear partial differential equations in three variables, Duke Math. J. vol. 13 (1946) pp. 419-458.

3. L. Kronecker, Zur Theorie der Elimination einer Variablen aus zwei algebraische Gleichungen, Berlin, 1881, pp. 535-600.

HARVARD UNIVERSITY AND

UNIVERSITY OF Notre DAME 